INPLASY

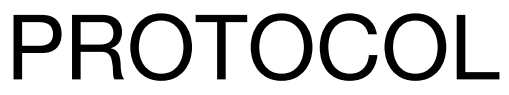

To cite: Tong et al. Platinum vs immunotherapy for early resectable non-small cell lung cancer: a systematic review and meta-analysis protocol. Inplasy protocol 202080064. doi:

10.37766/inplasy2020.8.0064

Received: 15 August 2020

Published: 15 August 2020

Corresponding author:

Zhangwei Tong

tongzhangwei123@163.com

Author Affiliation:

Fujian Medical University

Union Hospital

Support: JFNST:2018Y9034.

Review Stage at time of this submission: The review has not yet started.

Conflicts of interest:

The authors have no conflicts of interest to disclose.

\section{Platinum vs immunotherapy for early resectable non-small cell lung cancer: a systematic review and meta-analysis protocol}

Tong, Z1; Luo, F²; Yang, X3; Lin, J4.

Review question / Objective: Lung cancer is one of the most common malignant tumors. Non-small cell Lung cancer (NSCLC) accounts for about $85 \%$ of the total lung cancer. For patients with resectable early non-small cell lung cancer, conventional postoperative adjuvant therapy can significantly prolong the overall survival of patients and reduce the risk of tumor recurrence. With the emergence and maturity of molecular targeted therapy and immunotherapy, the strategy of postoperative chemotherapy for lung cancer patients has changed greatly. To evaluate the efficacy of postoperative chemotherapy (platinum based chemotherapy and immunotherapy) with or without radiotherapy for non-small cell lung cancer patients, we will conduct a systematic review and meta-analysis of published or unpublished randomized controlled trials.

INPLASY registration number: This protocol was registered with the International Platform of Registered Systematic Review and Meta-Analysis Protocols (INPLASY) on 15 August 2020 and was last updated on 15 August 2020 (registration number INPLASY202080064). 
strategy of postoperative chemotherapy for lung cancer patients has changed greatly. To evaluate the efficacy of postoperative chemotherapy (platinum based chemotherapy and immunotherapy) with or without radiotherapy for non-small cell lung cancer patients, we will conduct a systematic review and meta-analysis of published or unpublished randomized controlled trials.

Rationale: Patients with resectable early non-small cell lung cancer, conventional postoperative adjuvant therapy can significantly prolong the overall survival of patients and reduce the risk of tumor recurrence.

Condition being studied: Globally, lung cancer is the second most common malignancy. It is highly malignant and is the leading cause of cancer-related death[1]. Non small cell lung cancer (NSCLC) accounts for about $85 \%$ of the newly diagnosed lung cancer cases each year, which is the most common pathological type of lung cancer[2-3]. Surgical resection of early resectable non-small cell lung cancer offers potential therapeutic opportunities for patients considered to be the best treatment[4]. The 5-year survival rate of patients with pathological stage la to IIla NSCLC after surgery is $73-25 \%[5-6]$. However, there is no suitable treatment for the recurrence or metastasis of NSCLC. The 5-year survival rate of the patients can be increased by about $5 \%$ after the operation[7-9]. However, adjuvant radiotherapy is not recommended for postoperative adjuvant therapy of earlystage non-small cell lung cancer because it can significantly increase the mortality rate of patients by about $7 \%$ in 2 years[6,10-11]. With the emergence of molecular targeted therapy and immunotherapy, postoperative adjuvant therapy has become diversified[12]. At present, although there is not enough evidence to support that PD1/ PD-L1 inhibitors can effectively improve postoperative outcomes, more high-quality clinical trials are underway or have been published. Objective to evaluate the efficacy of postoperative chemotherapy.

\section{METHODS}

Search strategy: We will use the relevant keywords or subject terms adhered to Medical Subject Heading (MeSH) terms to search for eligible studies in the electronic databases which were mentioned above without language restrictions.

Participant or population: The participants will be adults diagnosed with resectable early NSCLC histologically or cytologically confirmed who were treated with platinumbased chemotherapy, or immunotherapy after surgery. No restrictions on ethnicity, sex, education, and economic status will be applied.

Intervention: According to the means of postoperative chemotherapy for patients with resectable early NSCLC, the trials included will be divided into the following categories. - immunotherapy versus molecular targeted therapy . immunotherapy versus anti-angiogenic agents - postoperative platinum-base chemotherapy versus molecular targeted therapy - platinum-based chemotherapy versus anti-angiogenic agents - platinumbased chemotherapy versus immunotherapy.

Comparator: We will evaluate the efficacy of postoperative adjuvant therapy (platinum based chemotherapy and immunotherapy) with or without radiotherapy for patients with NSCLC.

Study designs to be included: Randomized controlled trials (RCTs), quasi-RCTs, propensity score matched comparative studies and prospective cohort studies of interest.

Eligibility criteria: Eligibility criteria 3.1.1. Types of studies Randomised controlled trials (RCTs) and quasi-RCTs published or unpublished will be included, which have been completed and compared postoperative platinum-base chemotherapy versus immunotherapy for patients with NSCLC. 3.1.2. Types of participants The participants will be adults diagnosed with resectable early NSCLC 
histologically or cytologically confirmed who were treated with platinum-based chemotherapy, or immunotherapy after surgery. No restrictions on ethnicity, sex, education, and economic status will be applied. 3.1.3. Types of interventions According to the means of postoperative chemotherapy for patients with resectable early NSCLC, the trials included will be divided into the following categories. • immunotherapy versus molecular targeted therapy - immunotherapy versus antiangiogenic agents - postoperative platinum-base chemotherapy versus molecular targeted therapy - platinumbased chemotherapy versus antiangiogenic agents - platinum-based chemotherapy versus immunotherapy 3.1.4. Types of outcome measures Primary outcomes The primary outcomes will be postoperative overall survival of patients with resectable early NSCLC who were treated with postoperative chemotherapy. Secondary outcomes We will assess the 5year survival, median survival, recurrencefree survival, quality of life, and adverse events or complications of patients with resectable early NSCLC who were treated with postoperative chemotherapy.

Information sources: We will search Pubmed (Medline), Embase, Google Scholar, Cancerlit, and the Cochrane Central Register of Controlled Trials for related studies published before June 20, 2021 without language restrictions.

Main outcome(s): The primary outcomes will be postoperative overall survival of patients with resectable early NSCLC who were treated with postoperative chemotherapy.

Additional outcome(s): We will assess the 5-year survival, median survival, recurrence-free survival, quality of life, and adverse events or complications of patients with resectable early NSCLC who were treated with postoperative chemotherapy.

Data management: The two authors (ZWT, FL) will extract the following data independently from the studies included. •
Study characteristics and methodology: publication date, the first author, country, randomization, study design, periods of data collection, follow-up duration, total duration of study, and withdrawals, et al. • Participant characteristics: gender, age, tumor stage, pathology diagnosis, ethnicity, performance status, history of smoking, pathologic tumor size, and inclusion criteria, et al. - Interventions: type of operation, extent of resection, therapeutic means, drugs, dosage, modality and frequency of administration, et al. • Outcome and other data: overall survival, 5-year survival, median survival, diseasefree survival, $95 \%$ confidence intervals, recurrence time, quality of life, adverse events, and complications, et al. We will record all the date extracted in a predesigned table and consult the first author of the trial by e-mail before determining eligibility, if the reported data of which are unclear or missing.

Quality assessment / Risk of bias analysis: Two authors (ZWT, FL) will use the Cochrane Handbook for Systematic Reviews of Interventions to assess the risk of bias of each study included independently based on the following ranges: random sequence generation (selection bias); allocation concealment (selection bias); blinding of participants and personnel (performance bias); blinding of outcome assessment (detection bias); incomplete outcome data (attrition bias); selective outcome reporting(reporting bias); other bias[16]. Each domain will be assessed as high, low or uncertain risk of bias. The results and details of assessment will be reported on the risk of bias graph.

Strategy of data synthesis: The data will be synthesised by Review Manager 5.3 software. We will conduct a systematic review and meta-analysis only if the data gathered from included trials are judged to be similar enough to ensure a result that is meaningful. The Chi2 test and 12 statistic will be used to assess statistical heterogeneity among the trials included in matched pairs comparison for standard meta-analysis. The random effect model will be applied to analyse the data, if there 
is substantial heterogeneity (p50\%) and the trials will be regarded to be obvious heterogeneous. Otherwise, we will utilize fixed effect model to analyse the data. Mantel-Haenszel method will be adopted to pool of the binary data. The results will be reported in the form of relative risk (RR) between $95 \%$ confidence interval $(\mathrm{Cl})$ of the date. The continuous data will be pooled by inverse variance analysis method and the results will be shown in the form of standardized mean difference (SMD) with $95 \%$ confidence interval $(\mathrm{Cl})$ of the date.

Subgroup analysis: If there is high heterogeneity ( 12 statistic $>50 \%)$ and the data are sufficient, subgroup analysis will be conducted to search potential causes of heterogeneity. Subgroup analysis will be performed in different methods of postoperative adjuvant therapy, ethnicity, history of smoking, tumor stage, and type of operation.

Sensibility analysis: Sensitivity analysis will be conducted to assess the reliability and robustness of the aggregation results via eliminating trials with high bias risk.

\section{Language: Without any language restrictions.}

Country(ies) involved: China.

Keywords: Immunotherapy; radiotherapy; chemotherapy; non-small cell lung cancer; platinum chemotherapy.

Contributions of each author:

Author 1 - Zhangwei Tong - Author 1 drafted the manuscript.

Author 2 - Fei Luo - The author provided statistical expertise.

Author 3 - Xiaojie Yang - The author contributed to the development of the selection criteria, and the risk of bias assessment strategy.

Author 4 - Jiangbo Lin - The author read, provided feedback and approved the final manuscript. 